\title{
NEW CONSTRAINTS ON THE BLACK HOLE LOW/HARD STATE INNER ACCRETION FLOW WITH NuSTAR
}

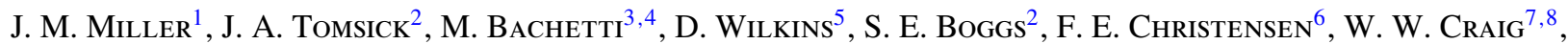 \\ A. C. Fabian $^{9}$, B. W. Grefenstette ${ }^{10}$, C. J. Hailey ${ }^{8}$, F. A. Harrison ${ }^{10}$, E. Kara ${ }^{9}$, A. L. King ${ }^{1}$, \\ D. K STERN ${ }^{11}$, AND W. W. ZHANG ${ }^{12}$ \\ ${ }^{1}$ Department of Astronomy, University of Michigan, 500 Church Street, Ann Arbor, MI 48109-1042, USA; jonmm@umich.edu \\ ${ }^{2}$ Space Sciences Laboratory, University of California, Berkeley, CA 94720, USA \\ ${ }^{3}$ Universite de Toulouse, UPS-OMP, IRAP, F-31100 Toulouse, France \\ ${ }^{4}$ CNRS, Intitut de Recherche in Astrophysique et Planetologie, 9 Av. Colonel Roche, BP 44346, F-31028 Toulouse, Cedex 4, France \\ 5 Department of Astronomy \& Physics, Saint Mary's University, Halifax, NS. B3H 3C3, Canada \\ ${ }^{6}$ Danish Technical University, DK-2800, Lyngby, Denmark \\ ${ }^{7}$ Lawrence Livermore National Laboratory, Livermore CA, USA \\ ${ }^{8}$ Columbia University, New York, NY 10027, USA \\ ${ }^{9}$ Institute of Astronomy, University of Cambridge, Madingley Road, Cambridge CB3 OHA, UK \\ ${ }^{10}$ Cahill Center for Astronomy and Astrophysics, California Institute of Technology, Pasadena, CA 91125, USA \\ ${ }^{11}$ Jet Propulsion Laboratory, California Institute of Technology, 4800 Oak Grove Drive, Pasadena, CA 91109, USA \\ 12 NASA Goddard Space Flight Center, Greenbelt, MD 20771, USA \\ Received 2014 September 2; accepted 2014 November 5; published 2015 January 19
}

\begin{abstract}
We report on an observation of the Galactic black hole candidate GRS 1739-278 during its 2014 outburst, obtained with NUSTAR. The source was captured at the peak of a rising "low/hard" state, at a flux of $\sim 0.3 \mathrm{Crab}$. A broad, skewed iron line and disk reflection spectrum are revealed. Fits to the sensitive NuSTAR spectra with a number of relativistically blurred disk reflection models yield strong geometrical constraints on the disk and hard X-ray "corona." Two models that explicitly assume a "lamp post" corona find its base to have a vertical height above the black hole of $h=5_{-2}^{+7} G M / c^{2}$ and $h=18 \pm 4 G M / c^{2}$ (90\% confidence errors); models that do not assume a "lamp post" return emissivity profiles that are broadly consistent with coronae of this size. Given that X-ray microlensing studies of quasars and reverberation lags in Seyferts find similarly compact coronae, observations may now signal that compact coronae are fundamental across the black hole mass scale. All of the models fit to GRS 1739-278 find that the accretion disk extends very close to the black hole-the least stringent constraint is $r_{\text {in }}=5_{-4}^{+3} G M / c^{2}$. Only two of the models deliver meaningful spin constraints, but $a=0.8 \pm 0.2$ is consistent with all of the fits. Overall, the data provide especially compelling evidence of an association between compact hard X-ray coronae and the base of relativistic radio jets in black holes.
\end{abstract}

Key words: accretion, accretion disks - black hole physics

\section{INTRODUCTION}

The phenomenon of relativistic radio jets in active galactic nuclei (AGNs) has long been appreciated, but the ability of black hole jets to shape host galaxies and even clusters of galaxies has only become clear recently (Fabian 2012). Accretion onto distant, low-luminosity, jet-producing AGNs is difficult to probe, however, owing to low X-ray flux levels. In contrast, Galactic stellar-mass black holes can provide very sensitive observations. In their low- $\dot{m}$, spectrally hard state ("low/hard" state), stellar-mass black holes launch compact, steady jets like those observed in some AGNs. If accretion flows are self-similar for given Eddington ratios, studies of Galactic black holes in the low/hard state may be the key to understanding jet production in AGNs.

GRS 1739-278 was discovered with the SIGMA telescope on board Granat (Paul et al. 1996; Vargas et al. 1997). The X-ray spectral and timing characteristics of GRS 1739-278 strongly suggest that the source harbors a black hole primary. In particular, a very strong $5 \mathrm{~Hz}$ quasi-periodic oscillation (QPO) was detected in GRS 1739-278, which is typical of black holes when they enter a "very high" state (Borozdin \& Trodolyubov 2000; Wijnands et al. 2001). Moreover, the source was detected in radio during its 1996 outburst (Hjellming et al. 1996), and strong radio emission in transients is typical of black holes; radio emission in neutron stars is notably weaker (Migliari \& Fender 2006). The Galactic center location of GRS 1739-278 and a measured extinction of $A_{V}=14 \pm 2$ strongly suggest that the source is located at a distance of 6-8.5 kpc (Dennerl \& Greiner 1996; Greiner et al. 1996).

Following an extended quiescent period, GRS 1739-278 was detected in outburst with the Swift Burst Alert Telescope (15-50 keV) on 2014 March 9 (Krimm et al. 2014). The source continued to brighten steadily, reaching a flux of approximately 0.065 counts s$^{-1}$ during its rise phase (the Crab gives a count rate of $\simeq 0.22$ counts $\mathrm{s}^{-1}$ ). The source was also noticed during monitoring observations of the Galactic center made with INTEGRAL; a hard $(\Gamma=1.4 \pm 0.2)$ cut-off power-law spectrum was detected out to $200 \mathrm{keV}$ (Filippova et al. 2014).

A prior NUSTAR (Harrison et al. 2013) observation of GRS 1915+105 in a "low/hard" (or, "plateau") state revealed a disk reflection spectrum in unprecedented detail (Miller et al. 2013). The sensitivity of that spectrum permitted a precise black hole spin measurement and indicated a small, centrally concentrated corona consistent with a "lamp post" geometry. NUSTAR has also measured black hole spin values in Cygnus X-1 and 4U 1630-472 (Tomsick et al. 2014; King et al. 2014). Motivated by these results, we triggered a NUSTAR observation of GRS 1739-278 to better explore black hole accretion flows in the jet mode. 

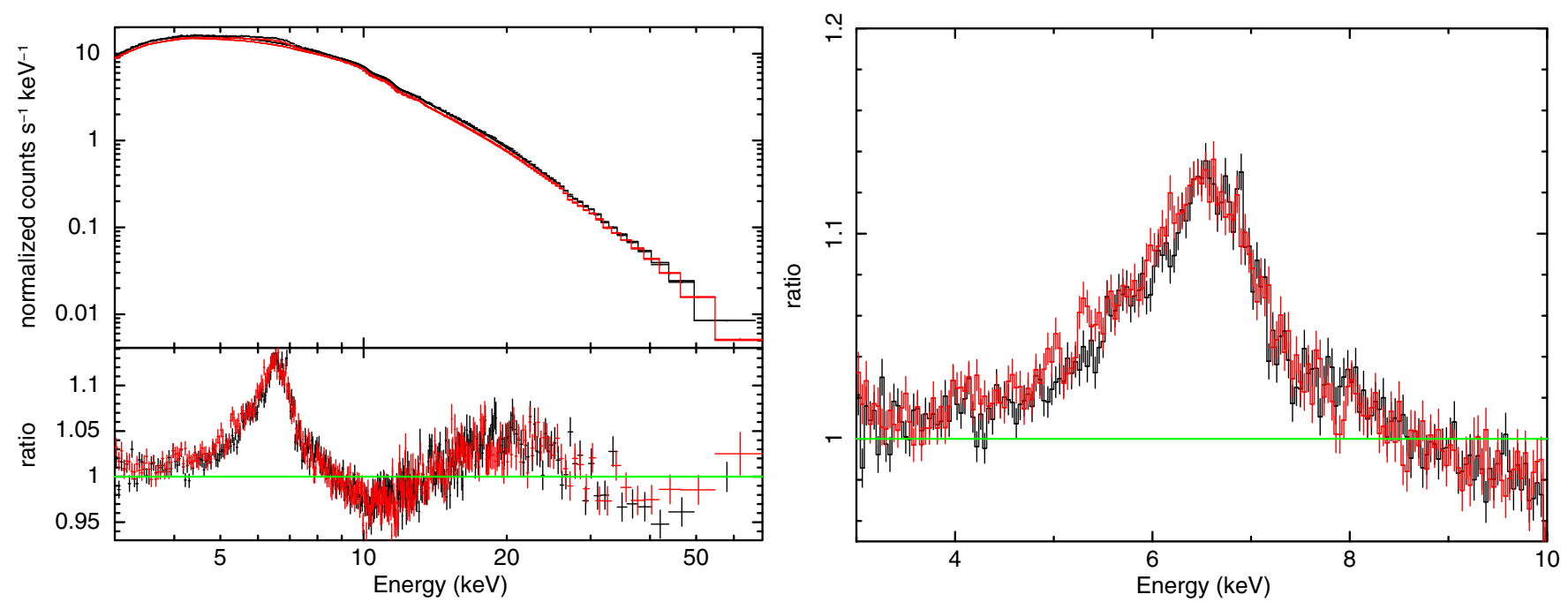

Figure 1. Simple cut-off power-law fits to the continuum emission in the NuSTAR spectrum of GRS 1739-278 (FPMA in black; FPMB in red). The left panel shows the data and ratio that result when the Fe $K$ band $(4-8 \mathrm{keV})$ is ignored while fitting the continuum. A skewed Fe K line and reflection "hump" are clearly visible in the spectra. The right panel shows the data/model ratio from the same fit, on a small linear scale in the Fe $K$ band. The breadth and characteristic shape of the relativistic line are clear. The $N_{\mathrm{H}}$ and $E_{\text {cut }}$ values in Table 1 were assumed in fitting the cut-off power-law continuum.

\section{DATA REDUCTION}

The NUSTAR observation of GRS 1739-278 started on 2014 March 26 at 16:06:07 UT. Event files and light curves were generated using the tools and routines in HEASOFT version 6.15.1, particularly NuSTARDAS version 1.3.1, and CALDB version 20131223. After filtering, the FPMA and FPMB detectors obtained net exposure times of $29.7 \mathrm{ks}$ and $30.4 \mathrm{ks}$, respectively.

Circular extraction regions with a radius of 120 arcsec, centered on the source position, were used to extract source counts. The very bright nature of the source likely provides no region on the focal plane that is truly free of source counts; regions of equivalent size but far from the source were extracted as background.

Prior to spectral fitting within XSPEC version $12.8 .1 \mathrm{~g}$ (Arnaud 1996), the spectra were grouped to require a signalto-noise ratio of at least 30 in all bins. This procedure exceeds the minimum quality necessary for $\chi^{2}$ statistical analysis to be valid (Cash 1979), but helps to build signal at high energy. All of the uncertainties quoted in this work are $90 \%$ confidence errors.

\section{ANALYSIS AND RESULTS}

Initial fits revealed some potential minor deficiencies in the response characterization. The highest energy bins lie slightly above plausible models; checks of the background show that these deviations are not likely to have a related origin. The problem was mitigated by ignoring the highest energy (grouped) bin. In our fits, then, the highest energy bins only extend up to $\sim 70 \mathrm{keV}$, rather than the nominal $79 \mathrm{keV}$. Similarly, small deviations were found at the lowest energies covered by FPMA and FPMB. In particular, the best fit line-of-sight column density in the FPMA spectrum is $N_{\mathrm{H}} \sim 2.3 \times 10^{22} \mathrm{~cm}^{-2}$; however, values lower by $0.3-0.4 \times 10^{22} \mathrm{~cm}^{-2}$ are preferred in the FPMB spectrum. Different source and background regions did not significantly alter this disparity. It is plausible that the difference results from small calibration errors. We therefore allowed the column density $N_{\mathrm{H}}$ to vary between the spectra, and, for simplicity, we only report the values measured for the FPMA detector. In view of these minor issues, we allowed the powerlaw index to float between the FPMA and FPMB detectors in all fits; however, any differences were found to be negligible, with $\Delta \Gamma \leqslant 0.02$. Last, we allowed an overall constant to float between the FPMA and FPMB to account for differences in their absolute flux calibrations.

Figure 1 shows the spectra of GRS 1739-278, fit with a simple cut-off power-law model, appropriate for black holes in the low/hard state (see Table 1). There is no evidence of thermal emission from an accretion disk; this likely owes to the low-disk temperatures typically measured in the low/hard state (e.g., $k T=0.2-0.3 \mathrm{keV}$; Miller et al. 2006; Reis et al. 2010; Reynolds \& Miller 2013), and a high level of line-ofsight obscuration.

Disk reflection features are clearly revealed in the data/model ratio in Figure 1. The rest of our analysis therefore focused on extracting information from the disk reflection spectrum. Reflionx is an established model that is suited to the high ionization levels expected in X-ray binaries (Ross \& Fabian 2005). In this work, an updated version of reflionx is used that includes a spectral cut-off in the illuminating power law as a fitting parameter (see, e.g., Miller et al. 2013). Fits were also made using the xillver reflection model (Garcia et al. 2013 , 2014), specifically xillver-a-Ec, which again includes an exponential cut-off. Xillver is relatively new; it features higher spectral resolution, newer atomic data, and includes angle dependencies. In contrast, reflionx is an "angle-averaged" model.

Reflection models are calculated in the fluid frame of the accretion disk, and a blurring function is required to translate from the fluid frame to the observed frame. These convolution models encode not only the relativistic Doppler shifts and gravitational red shifts expected close to black holes (ultimately allowing for spin measurements), but also the geometry of the hard X-ray corona. Whereas energy shifts encode the potential, the emissivity law captures the geometrical dependencies (see, e.g., Wilkins \& Fabian 2012; Dauser et al. 2013, 2014).

In this work, both reflionx and xillver were convolved with relconv to shift frames. The action of relconv on xillver is captured by the combined model relxill (Dauser et al. 2013). Xillver was also used in conjunction with two convolution models that assume a "lamp post" geometry (hard X-ray 
Table 1

Spectral Fits to GRS 1739-278

\begin{tabular}{|c|c|c|c|c|c|}
\hline & $\begin{array}{c}\text { relconv_lp_ext } \\
\times \text { xillyer }\end{array}$ & relxill_lp & relxill & $\begin{array}{l}\text { relconv } \\
\times \text { reflionx }\end{array}$ & $\begin{array}{l}\text { cut-off- } \\
\text { power law }\end{array}$ \\
\hline$N_{\mathrm{H}}\left(10^{22} \mathrm{~cm}^{-2}\right)$ & $2.18(6)$ & $2.35(6)$ & $1.94(6)$ & $1.95(5)$ & 4.6 \\
\hline$\Gamma$ & $1.46(1)$ & $1.47(1)$ & $1.44(1)$ & $1.44(1)$ & 1.65 \\
\hline$E_{\text {cut }}(\mathrm{keV})$ & $27.5(5)$ & $28.0(3)$ & 27.2(3) & $31.3(3)$ & 36 \\
\hline$K_{\text {power-law }}$ & $0.75(3)$ & $\ldots$ & & $0.658(5)$ & 1.3 \\
\hline$q_{\text {in }}$ & $\ldots$ & $\ldots$ & $8.5(8)$ & $8(1)$ & $\ldots$ \\
\hline$q_{\text {out }}$ & $\ldots$ & $\ldots$ & $2.0(1)$ & $1.9(1)$ & $\ldots$ \\
\hline$r_{\text {break }}\left(G M / c^{2}\right)$ & $\ldots$ & $\ldots$ & $6_{-0.1}$ & $6_{-0.1}$ & $\ldots$ \\
\hline$a\left(c J / G M^{2}\right)$ & $0(1)$ & $0(1)$ & $0.8(2)$ & $0.94(2)$ & $\ldots$ \\
\hline$r_{\text {in }}\left(G M / c^{2}\right)$ & $2_{-1}^{+5}$ & $5_{-4}^{+3}$ & $2.4(1)$ & $1.0^{+0.1}$ & $\ldots$ \\
\hline$h_{\text {corona }}\left(G M / c^{2}\right)$ & $5_{-2}^{+7}$ & $18(4)$ & $\ldots$ & $\ldots$ & $\ldots$ \\
\hline$v_{\text {corona }} / c$ & $0.98_{-0.98}^{+0.02}$ & $\ldots$ & $\ldots$ & $\ldots$ & $\ldots$ \\
\hline$i(\mathrm{deg})$ & $33(1)$ & $32.5(5)$ & $43.2(5)$ & $24(3)$ & $\ldots$ \\
\hline $\log (\xi)$ & $3.13(2)$ & $3.12(2)$ & $3.14(2)$ & $3.45(5)$ & $\ldots$ \\
\hline$A_{\mathrm{Fe}}$ & $1.8(2)$ & $1.5(2)$ & $1.9(2)$ & $0.61(3)$ & $\ldots$ \\
\hline$f_{\text {reflection }}$ & $\ldots$ & $0.174(4)$ & $0.18(2)$ & $\ldots$ & $\ldots$ \\
\hline$K_{\text {reflection }}$ & $1.07(7) \times 10^{-6}$ & $0.74(2)$ & $0.68(2)$ & $5.09(3) \times 10^{-6}$ & $\ldots$ \\
\hline$\chi^{2} / v$ & $1327.5 / 1105$ & $1330.3 / 1106$ & $1338.3 / 1105$ & 1339.3/1104 & $7869.7 / 1114$ \\
\hline
\end{tabular}

Notes. The results of a simple continuum fit (a cut-off power law), and with four different relativistically blurred disk reflection models are given in the table above (see the text for details). All quoted errors are $90 \%$ confidence limits. The normalization of different components is given in columns labeled with " $K$," $A_{\mathrm{Fe}}$ is the iron abundance relative to solar, and $f_{\text {refl }}$ is the reflection fraction.

emission from directly above the black hole, along its spin axis). The associated emissivity law is hardwired into these functions. Fits that assume a static "lamp post" corona were made using relxilllp, and fits exploring an outflowing "lamp post" with nonnegligible vertical extent were made by applying the function relconv_lp_ext to xillver.

The relxill and relconv* ${ }^{*}$ reflionx models require the addition of an external cut-off power law. In both cases, the power-law index and cut-off energy were linked between the reflection model and the external continuum component. These models do not assume a geometry for the corona, and therefore include an emissivity function encoded as a broken power law, giving parameters $q_{\text {in }}, q_{\text {out }}$, and $r_{\text {break }}$. Guided by the many different scenarios considered by Wilkins \& Fabian (2012), we required $3 \leqslant q_{\text {in }} \leqslant 10,0 \leqslant q_{\text {out }} \leqslant 3$, and $2 \leqslant c^{2} r_{\text {break }} / G M \leqslant 6$. In contrast, the fits made using relconv_lp_ext*xillver and relxilllp do not require emissivity indices, and instead constrain the height of the coronal base $h$ in units of $G M / c^{2}$. The relconv_lp_ext*xillver model is the most sophisticated of all; it essentially assumes that the corona is the base of a relativistic jet. It allows the data to constrain the vertical extent of the corona (not just the height), and also allows the data to constraint the velocity of the corona at its base and at its top. Preliminary tests suggested that the data could not constrain all of these parameters, so we only considered a corona with a single velocity ( $v_{\text {corona }} / c$ in Table 1$)$ and a fixed extent of $10 G M / c^{2}$.

Table 1 lists the results of fitting all four models, and Figure 2 shows the corresponding unfolded spectra and data/ model ratios. The models all measure similar power-law indices and values for the cut-off energy. The values of $N_{\mathrm{H}}$ are also broadly similar. This level of consistency likely signals that the continuum is well-measured, and not strongly model-dependent. Different reflection models give slightly different values for the ionization and abundances in the accretion disk. All three models based on xillver measure ionization values consistent with $\log (\xi)=3.12$, whereas the fit utilizing reflionx measures $\log (\xi)=3.45(5)$. The implied Fe abundances also differ between the models using xillver and relfionx.

All of the fits strongly suggest that the inner accretion disk in GRS 1739-278 remained close to the ISCO in this bright phase of the "low/hard" state. The least stringent constraint comes from fits with relxillp, which nominally allow for $r_{\text {in }}=8 \mathrm{GM} / \mathrm{c}^{2}$ at the $90 \%$ level of confidence. This is only slightly larger than the ISCO for prograde accretion onto an $a=0$ black hole. Figure 3 shows how the fit statistic varies for a larger range of possible inner disk radii. The best-fit model rules out a value of $r_{\text {in }} \sim 12 G M / c^{2}$ at the $5 \sigma$ level of confidence.

The models that explicitly assume a "lamp post" geometry measure small heights for the base of the corona: relconv_lp_ext*xillver gives $h=5_{-2}^{+7} G M / c^{2}$ (recall our model assumed a vertical extent of $10 G M / c^{2}$ above this base), and relxilllp gives $h=18 \pm 4 G M / c^{2}$. A very steep inner emissivity and flatter outer emissivity are commensurate with a "lamp post" emitter above a spinning black hole (Wilkins \& Fabian 2012; Dauser et al. 2013, 2014). The emissivity profiles measured from the other models in Table 1 are consistent with $q_{\text {in }}=8$ and $q_{\text {out }}=2$, indirectly indicating a lamp-post-like geometry with light bending close to a spinning black hole. These same models also provide strong direct constraints on the spin of the black hole, measuring high values consistent with $a=0.8 \pm 0.2$.

The models that explicitly assume a lamp post geometry do not provide meaningful constraints on the spin of the black hole in GRS 1739-278 (see Table 1 and Figure 3), though they certainly allow for high spin values. This may indicate potential degeneracies between geometric constraints and spin constraints in the "low/hard" state (Dauser et al. 2013, 2014; Fabian et al. 2014). In this sense, the spin measurements quoted above should be regarded with a degree of caution. Note, however, that although the lamp post models do not place strong constraints on the black hole spin, they still place strong constraints on the inner radius of the disk, ruling out large inner disk radii.

Both relxilllp and relxill indicate low values for the reflection fraction, much less than unity $\left(f_{\text {reflection }}=\right.$ reflected/incident $)$. 

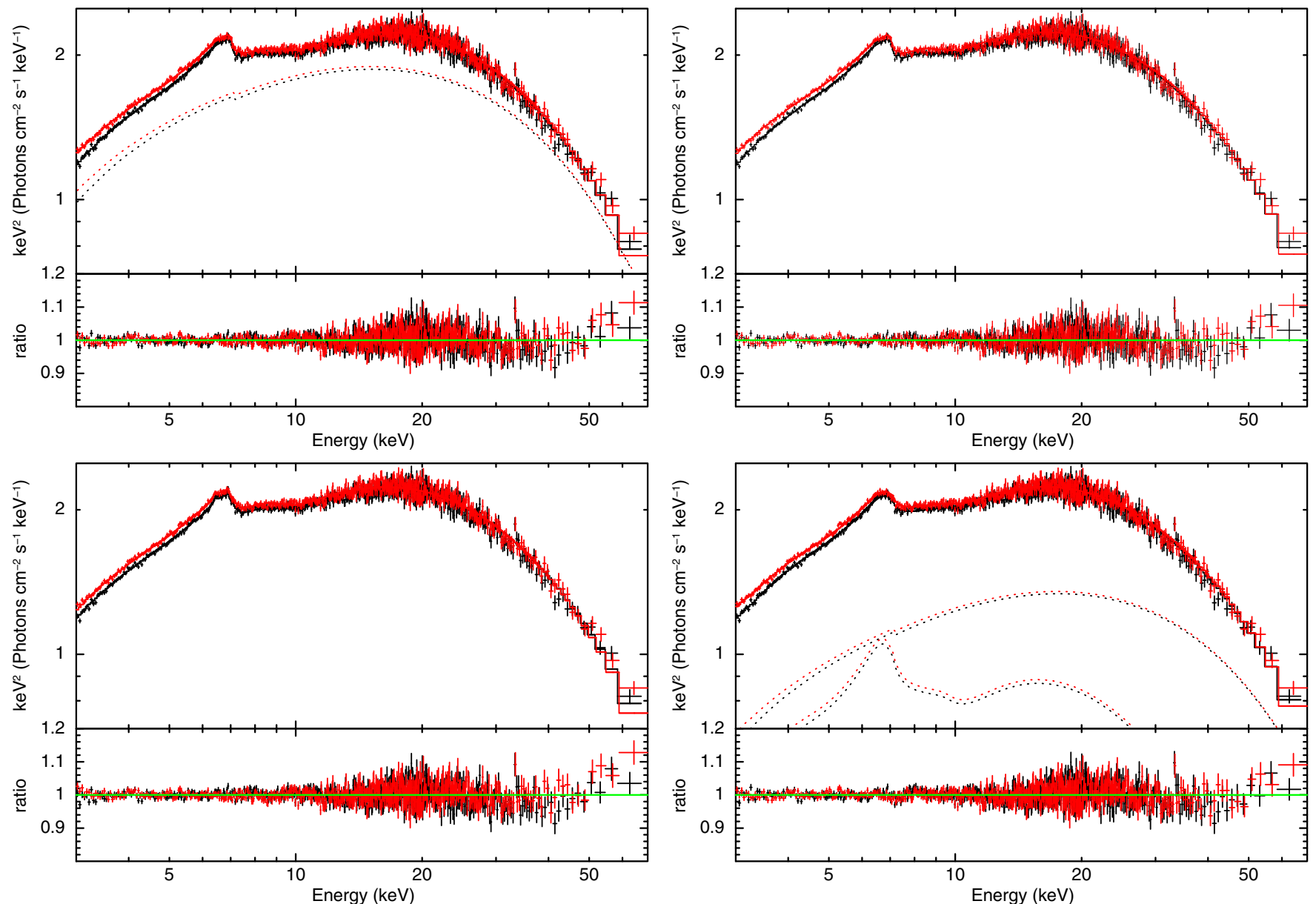

Figure 2. NUSTAR spectra of GRS 1739-278 are shown, fit with the relativistically blurred reflection models detailed in Table 1. In each panel, the FPMA spectrum is shown in black, and the FPMB spectrum in shown in red. Top left: relconv_lp_ext*xillver, the best overall fit. Top right: relxilllp, a close second-best fit. Bottom left: relxill, which does not assume a lamp post geometry. Bottom right: relconv*reflionx, which makes no geometrical assumption, and utilizes a different family of disk reflection models.
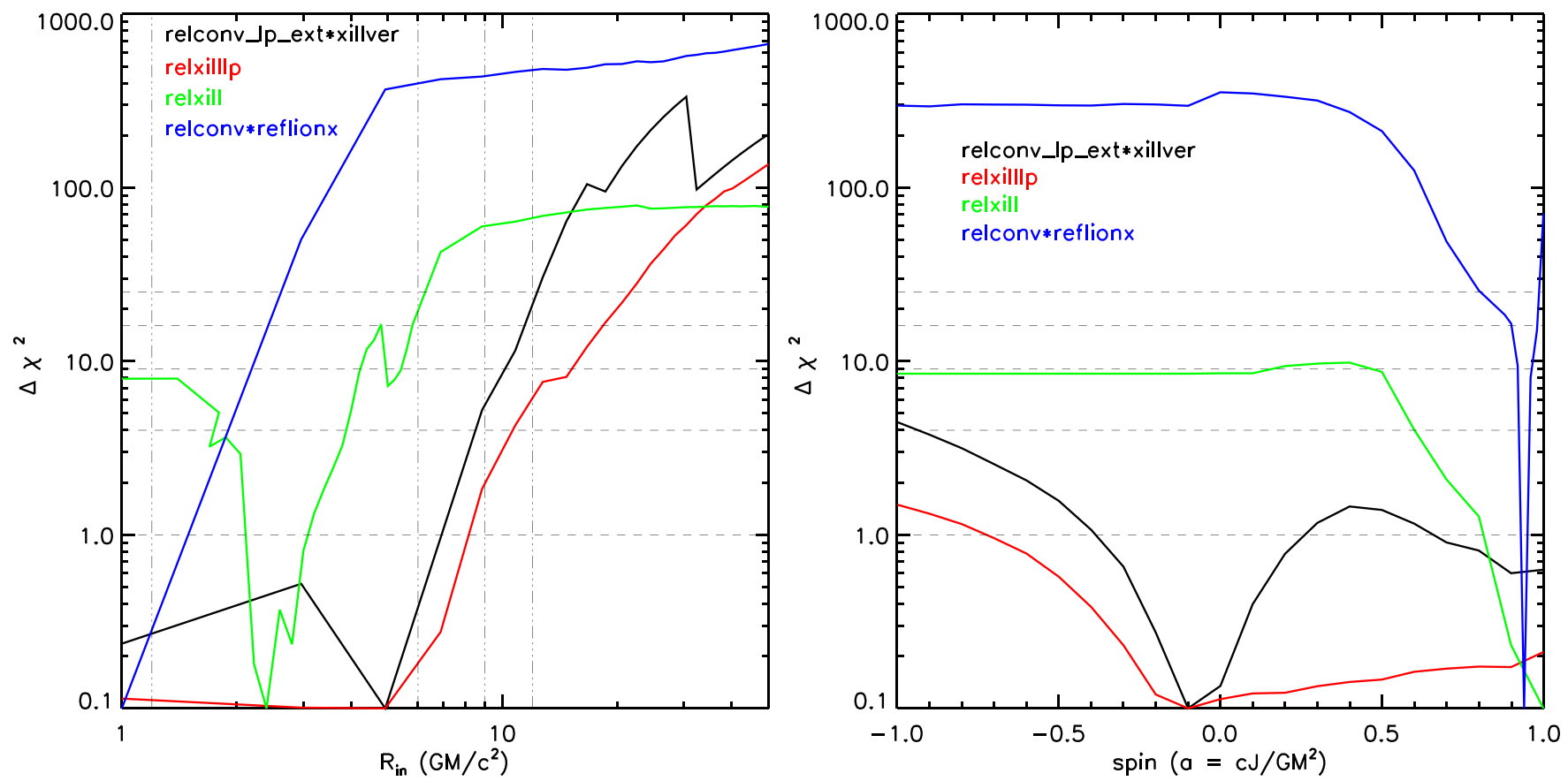

Figure 3. Confidence contours for key geometrical parameters of the relativistically blurred reflection models in Table 1. Left: the change in the goodness-of-fit statistic, $\chi^{2}$, is plotted vs. the inner disk radius. The dashed and dotted vertical lines indicate the ISCO radius for prograde and retrograde accretion disks, for both maximal and zero black hole spin. Right: the change in the goodness-of-fit statistic is plotted vs. the spin parameter of the black hole. In both panels, the dashed horizontal lines indicate the $1,2,3,4$, and $5 \sigma$ levels of confidence. 
This may be partly artificial: the models calculate the reflection fraction in the 20-40 keV band (Dauser et al. 2013, 2014). Extrapolating the fit with reflionx over the $0.001-1000 \mathrm{keV}$ band, a reflection fraction of $\sim 1.2$ is obtained. If the low fraction is physical, it could be explained via a truncated accretion disk that does not subtend the expected solid angle, or a corona that has beamed part of its emission away from the disk, or a stratified disk atmosphere that diminishes the overall reflection spectrum (Ballantyne \& Fabian 2001; Nayakshin \& Kallman 2001). Given that all of the models strongly require small inner disk radii and small coronae, the low reflection fraction may indicate an outflowing corona, as predicted if the corona is the base of a relativistic jet (Markoff et al. 2005; also see Miller et al. 2006, 2013). Indeed, the best-fit model in Table 1 is relconv_lp_ext*xillver, which assumes an outflowing corona (note, however, that the data are not able to constrain the velocity of the outflow).

Finally, we attempted to explicitly examine whether or not the data can reject a radially extended corona. Wilkins \& Fabian (2012) give different emissivity prescriptions, corresponding to different coronal geometries. Their work captures the emissivity in terms of two break radii, whereas a model like relconv only has one break. Based on the best fit to 1H 0707-495 detailed in Wilkins \& Fabian (2012), fake spectra were generated assuming $q_{\text {in }}=7.8, r_{\text {break }}=5 G M / c^{2}, q_{\text {mid }}=0$, and $q_{\text {out }}=3.3$, but varying the outer break radius $\left(J \propto r^{-q}\right)$. The fake spectra were then fit with an emissivity profile that has only one break, in order to establish how a simpler emissivity prescription might encode a radially extended corona.

A "true" coronal radial extent of $50 \mathrm{GM} / \mathrm{c}^{2}$ is encoded as having $q_{\text {in }}=5.09, q_{\text {out }}=1.73$, and $r_{\text {break }}=5.09 \mathrm{GM} / \mathrm{c}^{2}$ when only a single break is used. Fits to GRS 1739-278 with the relxill model give $\chi^{2}=1353.0 / 1107$ for this emissivity prescription (all other parameters were free to vary). The best-fit "lamp post" model is preferred over this example of a radially extended corona at the $4.2 \sigma$ level of confidence. However, the simplest geometry with a truncated disk is one wherein the corona lies entirely within the truncated disk. This corresponds to an ADAF-like geometry in which the inner disk has become too hot to be cool and thin. If the inner radius is fixed to the (encoded) break radius of $5.09 \mathrm{GM} / \mathrm{c}^{2}$, the fit becomes even worse: $\chi^{2} / \nu=1400.5 / 1108$. A "lamp post" geometry is preferred to this prescription at the $7.1 \sigma$ level of confidence.

\section{DISCUSSION AND CONCLUSIONS}

We have analyzed spectra of the transient and recurrent black hole candidate GRS 1739-278, obtained near the peak of a rising "low/hard" state with NUSTAR. The extraordinary sensitivity and broad energy range of the spectra reveal a reflection spectrum in detail, and strong geometric constraints are derived. The data strongly signal reflection from an accretion disk that remains close to the black hole, irradiated by a compact and potentially outflowing corona at a moderate height above the black hole. Compared to "very high" or "intermediate" states, then, the peak of the "low/hard" state may be characterized by weaker illumination of the disk (perhaps due to beaming of coronal/jet emission), and a larger coronal scale height. In this section, we review these results and discuss their implications.

The results listed in Table 1 and shown in Figure 3 indicate that the disk in GRS 1739-278 remained close to plausible ISCO values. Sensitive NuSTAR spectra of the persistent stellar-mass black hole GRS 1915+105 in a "low/hard" state also required a disk extending to the ISCO around a rapidly spinning black hole (Miller et al. 2013). Moreover, a series of 20 Suzaku observations of the archetypical black hole Cygnus X-1 in the "low/hard" state revealed a blurred disk reflection spectrum that requires a disk that extends close to the ISCO (Miller et al. 2012a); Fabian et al. (2012) used these data to measure a spin of $a=0.97_{-0.02}^{+0.01}$ and an inner radius of $r_{\text {in }} \leqslant 2 G M / c^{2}$.

Esin et al. (1997) predicted that disks may truncate at $\dot{m} \leqslant 0.08$, corresponding to $L / L_{\text {Edd }} \leqslant 0.008$ (assuming an efficiency of $\eta=0.1)$. The best-fit model for GRS 1739-278 in Table 1 implies $L=1.0 \times 10^{38} \mathrm{erg} \mathrm{s}^{-1}(0.5-100.0 \mathrm{keV})$ or $L / L_{\text {Edd }} \simeq 0.08$ for a black hole of $10 M_{\odot}$. Indeed, all of the results cited above were obtained in luminous phases of the "low/hard" state, above the predicted truncation luminosity. Our results simply indicate, then, that state transitions - and jet production-are driven primarily by coronal changes, rather than large or rapid changes in the disk. This may also be supported by relatively smooth variations in disk temperatures across state transitions (e.g., Rykoff et al. 2007; also see Gierlinski et al. 2009). This only requires that $\dot{M}$ varies while $R$ remains relatively constant, since the disk temperature and viscous dissipation depend on both $\dot{M}$ and $R$. Deeper into the "low/hard" state, $\dot{M}$ and $R$ might decrease together. Positive evidence of disk truncation at $L / L_{\text {Edd }} \simeq 0.001$ was indicated by a narrow Fe K line in a Suzaku spectrum of GX 339-4 (Tomsick et al. 2009).

The reflection fits also provide important indications for a very compact, and potentially outflowing corona that is likely the base of a relativistic jet. The small coronal heights that are measured with two models, the steep emissivity profiles measured with two others, small measured reflection fractions (potentially), and the fact that the best overall model assumes an outflowing corona, all point in this direction. X-ray reverberation from the inner disk in Seyfert AGNs strongly indicates short distance scales and small coronal heights (e.g., Zoghbi et al. 2010; De Marco et al. 2013). Also, groundbreaking X-ray microlensing measurements in quasars also constrain the hard $\mathrm{X}$-ray corona to have a size of only $\simeq 10-20 \mathrm{GM} / \mathrm{c}^{2}$ (e.g., Morgan et al. 2010; Blackburne et al. 2014). It is possible, then, that compact coronae are a central, mass-independent feature of black hole accretion and jet production.

Coronal energetics also point toward an association with the base of a jet. Merloni \& Fabian (2001) considered the coronal scales necessary to match implied X-ray luminosities, assuming that $\mathrm{X}$-ray coronae are powered by pure thermal Comptonization. Using the cut-off energy values given in Table 1 , and further assuming $\tau \simeq 0.1$, the corona in GRS $1739-278$ would need to be $10^{4} G M / c^{2}$ in radial extent to generate the implied luminosity. Even $\tau \simeq 1$ gives $10^{3} \mathrm{GM} / \mathrm{c}^{2}$. The NUSTAR spectra do not allow for a hole of this radius within the disk or a spheroid of such a height. Based on such contradictions, and the small length scales implied by X-ray variability, Merloni \& Fabian (2001) found that coronae must be primarily nonthermal, and likely magnetic. A broad range of models predict that magnetic fields play a role in jet production. Very compact coronae may hint at close interactions with black holes, as per Blandford \& Znajek (1977).

J.M.M. thanks Javier Garcia and Thomas Dauser for helpful conversations. This work was supported under NASA Contract No. NNG08FD60C, and made use of data from the NuSTAR mission, a project led by the California Institute of Technology, managed by the Jet Propulsion Laboratory, and funded by NASA. 


\section{REFERENCES}

Arnaud, K. 1996, in Astronomical Data Analysis Software and Systems V, in ASP Conf. Ser. 101, ed. G. H. Jacoby \& J. Barnes (San Francisco, CA: ASP), 17

Ballantyne, D., \& Fabian, A. C. 2001, MNRAS, 327, 10

Blackburne, J., Kochanek, C., Chen, B., Dai, X., \& Chartas, G. 2014, ApJ, 789,125

Blandford, R. D., \& Znajek, R. L. 1977, MNRAS, 179, 433

Borozdin, K., \& Trudolyubov, S. P. 2000, ApJL, 533, L131

Cash, W. 1979, ApJ, 228, 939

Dauser, T., Garcia, J., Wilms, J., et al. 2013, MNRAS, 430, 1694

Dauser, T., Garcia, J., Parker, M. L., Fabian, A. C., \& Wilms, J. 2014, MNRAS, 444, L100

Dennerl, K., \& Greiner, J. 1996, IAU Circ., 6426

De Marco, B., Ponti, G., Cappi, M., et al. 2013, MNRAS, 431, 2441

Esin, A. A., McClintock, J. E., \& Narayan, R. 1997, ApJ, 489, 865

Fabian, A. C. 2012, ARA\&A, 50, 455

Fabian, A. C., Parker, M. L., Wilkins, D. R., et al. 2014, MNRAS, 439, 2307

Fabian, A. C., Wilkins, D. R., Miller, J. M., et al. 2012, MNRAS, 424, 217

Filippova, E., Kuulkers, E., Skådt, N. M., et al. 2014, ATel, 5991

Garcia, J., Dauser, T., Lohfink, A., et al. 2014, ApJ, 782, 76

Garcia, J., Dauser, T., Reynolds, C. S., et al. 2013, ApJ, 768, 146

Gierlinski, M., Done, C., \& Page, K. 2009, MNRAS, 392, 1106

Greiner, J., Dennerl, K., \& Predehl, P. 1996, A\&A, 314, L21
Harrison, F. A., Craig, W. W., Christensen, F. E., et al. 2013, ApJ, 770, 103 Hjellming, R. M., Rupen, M. P., Marti, J., Mirabel, F., \& Rodriguez, L. F. 1996, IAU Circ., 6383

King, A. L., Walton, D. J., Miller, J. M., et al. 2014, ApJL, 784, L2

Krimm, H., Barthelmy, S. D., Baumgartner, W., et al. 2014, ATel, 5986

Markoff, S., Nowak, M., \& Wilms, J. 2005, ApJ, 635, 1203

Merloni, A., \& Fabian, A. C. 2001, MNRAS, 321, 549

Miller, J. M., Pooley, G. G., Fabian, A. C., et al. 2012a, ApJ, 757, 11

Miller, J. M., Parker, M. L., Fuerst, F., et al. 2013, ApJL, 775, L45

Miller, J. M., Raymond, J., Homan, J., et al. 2006, ApJ, 646, 394

Migliari, S., \& Fender, R. P. 2006, MNRAS, 366, 79

Morgan, C. W., Kochanek, C. S., Morgan, N. D., \& Falco, E. E. 2010, ApJ, 712,1129

Nayakshin, S., \& Kallman, T. 2001, ApJ, 546, 406

Paul, J., Bouchet, L., Churazov, E., \& Sunyaev, R. 1996, IAU Circ., 6348

Reis, R. C., Fabian, A. C., \& Miller, J. M. 2010, MNRAS, 402, 836

Reynolds, M., \& Miller, J. M. 2013, ApJ, 769, 16

Ross, R., \& Fabian, A. C. 2005, MNRAS, 358, 211

Rykoff, E., Miller, J. M., Steeghs, D., \& Torres, M. A. P. 2007, ApJ, 666, 1129

Tomsick, J. A., Yamaoka, K., Corbel, S., et al. 2009, ApJL, 707, L87

Tomsick, J. A., Nowak, M. A., Parker, M., et al. 2014, ApJ, 780, 78

Vargas, M., Goldwurm, A., Laurent, P., et al. 1997, ApJL, 476, L23

Wijnands, R., Mendez, M., Miller, J. M., \& Homan, J. 2001, MNRAS, 328, 451

Wilkins, D. R., \& Fabian, A. C. 2012, MNRAS, 424, 1284

Zoghbi, A., Fabian, A. C., Uttley, P., et al. 2010, MNRAS, 401, 2419 